\title{
Fine mapping of a quantitative trait locus for bovine milk fat composition on Bos taurus autosome 19
}

\author{
Aniek C. Bouwman, ${ }^{1}$ Marleen H. P. W. Visker, Johan A. M. van Arendonk, and Henk Bovenhuis \\ Animal Breeding and Genomics Centre, Wageningen University, PO Box 338, 6700 AH Wageningen, the Netherlands
}

\begin{abstract}
A major quantitative trait locus (QTL) for milk fat content and fatty acids in both milk and adipose tissue has been detected on Bos taurus autosome 19 (BTA19) in several cattle breeds. The objective of this study was to refine the location of the QTL on BTA19 for bovine milk fat composition using a denser set of markers. Opportunities for fine mapping were provided by imputation from 50,000 genotyped single nucleotide polymorphisms (SNP) toward a high-density SNP panel with up to 777,000 SNP. The QTL region was narrowed down to a linkage disequilibrium block formed by 22 SNP covering $85,007 \mathrm{bp}$, from $51,303,322$ to $51,388,329 \mathrm{bp}$ on BTA19. This linkage disequilibrium block contained 2 genes: coiled-coil domain containing 57 (CCDC57) and fatty acid synthase $(F A S N)$. The gene $C C D C 57$ is minimally characterized and has not been associated with bovine milk fat previously, but is expressed in the mammary gland. The gene FASN has been associated with bovine milk fat and fat in adipose tissue before. This gene is a likely candidate for the QTL on BTA19 because of its involvement in de novo fat synthesis. Future studies using sequence data of both CCDC57 and $F A S N$, and eventually functional studies, will have to be pursued to assign the causal variant(s).
\end{abstract}

Key words: Bos taurus autosome 19, fine mapping, milk fatty acid, quantitative trait loci

\section{INTRODUCTION}

Many linkage and genome-wide association studies (GWAS) have been performed to identify QTL in cattle. These studies have detected numerous chromosomal regions affecting traits of interest (e.g., http://www. animalgenome.org/cgi-bin/QTLdb/index; Khatkar et al., 2004; Hu et al., 2010). Typically, the location of the QTL is estimated inaccurately and the confidence interval contains several candidate genes. To fine-map QTL, researchers have genotyped additional markers

Received June 29, 2013.

Accepted October 13, 2013.

${ }^{1}$ Corresponding author: Aniek.Bouwman@wur.nl on the same animals, genotyped additional animals, and sometimes sequenced candidate genes (e.g., Grisart et al., 2002; Meuwissen et al., 2002; Blott et al., 2003; Cohen-Zinder et al., 2005; Gautier et al., 2006; Druet et al., 2008; Kim et al., 2009; Karim et al., 2011). Currently, opportunities for fine-mapping QTL in cattle are provided by the availability of high-density SNP panels with up to 777,000 SNP. Genotyping at higher density, or imputation of genotypes to higher densities, increases the power of GWAS, gives a more detailed view of associated regions, and increases the chance of one of the SNP being in strong linkage disequilibrium (LD) with the causal variant of the QTL (Marchini et al., 2007; Spencer et al., 2009; Marchini and Howie, 2010).

For milk FA, a few genome-wide linkage studies and GWAS have been performed. Those studies showed that 3 regions exist with major effects on milk FA, located on BTA14, BTA19, and BTA26 (Schennink et al., 2009b; Stoop et al., 2009; Bouwman et al., 2011, 2012). The region on BTA14 has been studied extensively and a dinucleotide polymorphism in diacylglycerol-Oacyltransferase 1 (DGAT1) has been suggested as the causal variant (Grisart et al., 2002; Schennink et al., 2007). For the region on BTA26, a polymorphism in stearoyl-CoA desaturase 1 (SCD1) has been suggested as causal variant (Taniguchi et al., 2004; Schennink et al., 2008). For the region on BTA19, the causal variant has not been identified.

The QTL on BTA19 shows association with several FA in both milk and adipose tissue (Morris et al., 2007; Bouwman et al., 2011, 2012; Ishii et al., 2013). Fatty acid synthase $(\boldsymbol{F A} \boldsymbol{S N})$ has been suggested as a candidate gene and several SNP in FASN are significantly associated with FA in both beef and dairy cattle (Roy et al., 2006; Morris et al., 2007; Ordovás et al., 2008; Zhang et al., 2008; Abe et al., 2009; Schennink et al., 2009a; Oh et al., 2012), but the QTL region is rather large and shows much higher significance levels than those observed in the candidate gene studies (Bouwman et al., 2011). Therefore, the objective of this study was to refine the location of the QTL on BTA19 for bovine milk fat composition previously reported by Bouwman et al. (2011), using a denser set of markers. 


\section{MATERIALS AND METHODS}

\section{Population}

Detailed fat composition was measured in milk samples from 1,905 first-lactation Dutch HolsteinFriesian cows, which were housed on 398 commercial farms throughout the Netherlands. At least 3 cows were sampled per herd. Milk samples were taken in winter (February to March 2005), when Dutch cows are mainly kept indoors and fed silage. The cows were between 63 and 282 DIM at the day of sampling. About half of the sampled cows were descendants from 5 proven sires (101-200 daughters per sire); the other half of the sampled cows descended from 50 test sires or 45 other sires (1-30 daughters per sire). The pedigree of the cows was provided by the Cooperative Cattle Improvement Organization (CRV, Arnhem, the Netherlands) and consisted of 26,300 animals.

\section{Phenotypes}

Milk FA were measured by gas chromatography and were expressed in terms of weight-proportion of total milk fat weight (wt/wt\%). More details about the phenotypes can be found in Stoop et al. (2008).

This study focuses on C14:0, because in previous studies, this milk FA showed the strongest association with the region on BTA19 (Morris et al., 2007; Stoop et al., 2009; Bouwman et al., 2011). Milk fat of the 1,905 cows contained, on average, $11.61 \% \mathrm{C} 14: 0$, the phenotypic standard deviation of C14:0 was 0.78 , the heritability of C14:0 was 0.62 , and the QTL on BTA19 explained approximately $13.8 \%$ of the genetic variation in C14:0 (Bouwman et al., 2011).

\section{Genotypes}

Initially, 1,810 cows and 55 of their sires (all proven and test sires) were genotyped with a custom 50,000 (50K)-SNP array (Illumina Inc., San Diego, CA) designed by CRV. The 55 sires were regenotyped using the BovineHD BeadChip (Illumina Inc.) with 777,000 $(\mathbf{7 7 7 K})$ SNP. The high-density (HD) genotypes of these 55 sires were combined with HD genotypes of other Dutch Holstein-Friesians available at CRV to form a reference population for imputation of, in total, 1,333 HD genotyped Dutch Holstein-Friesian animals.

Animals with pedigree inconsistencies (71 cows) were removed before imputation. Pedigree inconsistencies were assumed when more than $0.5 \%$ of the $50 \mathrm{~K}$ SNP for which both sire and daughter were homozygous, were homozygous for the opposite allele. The software BEAGLE 3.3 (Browning and Browning, 2009) was used to phase and impute missing genotypes for the HD reference animals. These phased genotypes were then used to impute the $50 \mathrm{~K}$ genotypes of the cows to HD genotypes. The assumed map positions of the SNP were based on the bovine genome assembly UMD 3.1 (Zimin et al., 2009).

In the $50 \mathrm{~K}$ data, 1,454 SNP were located on BTA19, with an average distance of 44,888 bp. The number of SNP on BTA19 increased to 18,893 , using the HD imputed data, with an average distance between SNP of 3,386 bp. We found 998 SNP overlapping between the 50K SNP panel and the HD SNP panel. A total of 1,572 monomorphic SNP in the HD-imputed data were excluded, and in addition 2,659 SNP were excluded because they had a low genotype frequency (i.e., 1-9 individuals within 1 of the 3 genotype classes), resulting in 14,662 SNP used in this study.

\section{Single SNP Association Analysis}

For 1,640 cows with both C14:0 phenotypes and HD-imputed genotypes, a single SNP analysis was performed for BTA19 using the following mixed model in ASReml software (VSN International Ltd., Hemel Hempstead, UK):

$y_{i j k l m n o}=\mu+b_{1} \times \operatorname{dim}_{i}+b_{2} \times e^{-0.05 \times \operatorname{dim}_{i}}+b_{3} \times \operatorname{afc}_{j}+b_{4} \times \operatorname{afc}_{j}^{2}$
$+\operatorname{season}_{k}+\operatorname{scode}_{1}+\operatorname{herd}_{m}+\operatorname{genotype}_{n}+\operatorname{animal}_{\mathrm{o}}+e_{i j k l m n o}$,

where $y_{\mathrm{ijklmno}}$ was the phenotype; $\mu$ was the overall mean; $b_{1}$ to $b_{4}$ were regression coefficients of corresponding covariates; $\operatorname{dim}_{\mathrm{i}}$ was the covariate describing the effect of DIM; afc $\mathrm{c}_{j}$ was the covariate describing the effect of age at first calving; $\operatorname{season}_{k}$ was the class variable accounting for calving season (June-August 2004, September-November 2004, or December 2004-January 2005); scode was $_{1}$ the class variable accounting for differences in genetic level between proven-sire daughters and test-sire daughters; herd $\mathrm{m}_{\mathrm{m}}$ was the random effect of herd, distributed as $\mathrm{N}\left(0, \mathbf{I} \sigma_{\text {herd }}^{2}\right)$, with identity matrix I and herd variance $\sigma_{\text {herd }}^{2}$; genotype was $_{\text {ne }}$ class variable accounting for the genotype of the SNP; animal $_{\circ}$ was the random additive genetic effect, distributed as $\mathbf{N}\left(0, \mathbf{A} \sigma_{\mathrm{a}}^{2}\right)$, with additive genetic relationship matrix $\mathbf{A}$ based on the full pedigree and additive genetic variance $\sigma_{\mathrm{a}}^{2}$; and $\mathrm{e}_{\mathrm{ijklmno}}$ was the random residual, distributed as $\mathrm{N}\left(0, \mathbf{I} \sigma_{\mathrm{e}}^{2}\right)$, with identity matrix $\mathbf{I}$ and residual variance $\sigma_{\mathrm{e}}^{2}$. To speed up the single SNP association analysis of all 14,662 SNP on BTA19, the genetic and herd variances were fixed to the variances estimated using model 1 without the genotype effect. 
A significance threshold was calculated using a Bonferroni-like correction for multiple testing according to the method of Šidák (1967): $1-(1-\alpha)^{1 / n}$, where $\mathrm{n}$ is the number of SNP (here, 14,662) and $\alpha$ is the significance level (here, $0.1 \%$ ). This rather conservative threshold was chosen because the main interest of this study was reducing false positives, whereas missing false-negative associations was of less concern because we have previously shown highly significant evidence for the presence of a QTL on BTA19 (Bouwman et al., 2011, 2012), which has also been confirmed in other cattle populations (Morris et al., 2007; Ishii et al., 2013).

The genetic variance explained by a SNP was calculated from the estimated genotype effects and the observed genotype frequencies. The result was expressed as a percentage of the total additive genetic variance obtained from model 1 without the genotype effect.

The $\mathrm{R}$ package biomaRt (Durinck et al., 2009) was used on all 14,662 SNP to determine if SNP were located in genes and, if so, in which genes they were located. For the SNP mentioned in the tables, the Basic Local Alignment Search Tool (BLAST; http://blast. ncbi.nlm.nih.gov/Blast.cgi) was used to compare the SNP sequences with the bovine genome sequence to confirm the location of the SNP in the genes and to infer the functional consequences of the SNP.

\section{Haplotype Analysis}

The imputation in BEAGLE resulted in phased genotypes for all individuals, which provided the opportunity to study haplotypes. Haplotypes better characterize a chromosomal segment than any single SNP. This is relevant when the causal variant is not one of the typed SNP or in full LD with any of the typed SNP. In such a case, a haplotype might capture more LD with the causal variant and, consequently, provide a better estimate of the effect of the causal variant than any of the typed SNP. When the haplotype characterizes the chromosomal segment that contains the causal variant and the causal variant is not one of the typed SNP or in full LD with any of the typed SNP, the effect of the haplotype should be larger than the effect of any of the typed SNP. Two different types of haplotype analysis were performed and are described in more detail in the following section: (1) sliding window of 2 consecutive SNP and (2) LD block analysis.

Sliding Window. For the 1,640 cows (with both genotypes and phenotypes), phased genotypes of 2 neighboring SNP were used to create 2-SNP haplotypes, and the 2 haplotypes per cow were combined into a genotype. The genotypes of the 2-SNP haplotypes were included as a class variable in model 1 instead of the single SNP genotype. This association analysis was used to screen the whole chromosome 19 with a sliding window of 2 consecutive SNP, shifting 1 SNP at a time.

LD Block. Haploview 4.2 software (Barrett et al., 2005) was used to define LD blocks for the region between 51.2 and $51.5 \mathrm{Mbp}$ on BTA19 that contained the most significant SNP from both the single SNP and the sliding-window analyses. The LD blocks were defined using all $84 \mathrm{SNP}$ in the region and all 1,640 cows (with both phenotypes and genotypes). The LD blocks were based on the $\mathrm{D}^{\prime}$ measure of LD (default Haploview options).

Six LD blocks were defined in the region, of which LD block 3 contained the most significantly associated SNP in both the single SNP and the sliding-window analyses. Therefore, LD block 3 was studied in more detail. Nine haplotypes within LD block 3 had reasonable frequencies $(>1 \%)$ and were tested for association with C14:0. For 1 of the 9 haplotypes at a time, the SNP genotype in model 1 was replaced by the number of copies of the haplotype tested $(0,1$, or 2$)$ as a covariable. This association analysis was repeated for each of the 9 haplotypes.

\section{Correction for Most Significant SNP and 2-SNP Window}

To test whether multiple QTL were located on BTA19, the single SNP association analysis was repeated for C14:0 phenotypes $(\mathbf{y})$ corrected for the genotype effects of (a) the most significant SNP of the single SNP analysis and (b) the most significant 2-SNP window of the sliding-window analysis. In both cases, BTA19 was screened using a single SNP analysis as described above, using model 1 on the precorrected phenotypes $\left(\mathbf{y}^{*}\right)$, where $\mathbf{y}^{*}=\mathbf{y}-\mathbf{X}_{\text {geno }} \mathbf{b}$, with $\mathbf{X}_{\text {geno }}$ representing an incidence matrix for the genotype of (a) the most significant SNP of the single SNP analysis or (b) the most significant 2-SNP window of the sliding-window analysis and $\mathbf{b}$ representing a vector with the estimated effect of each genotype.

\section{RESULTS}

\section{Single SNP in CCDC57 Most Significantly Associated With C14:0}

Figure 1 shows the significance of association between C14:0 and 14,662 SNP on BTA19, resulting from a chromosome-wide single SNP association analysis. Imputed SNP showed a stronger signal than empirically genotyped SNP, but the most significant empirically genotyped SNP was located in the same region as the most significant imputed SNP (Figure 1). Based 
on the Bonferroni threshold with $\alpha=0.1 \%\left[-\log _{10}(P\right.$ value $)=7.17$, there were 284 significant SNP, which were located between 40,228,233 and 58,072,578 bp. Within this part of the chromosome, 3 regions were most pronounced: around $42.4 \mathrm{Mbp}$, around $51.3 \mathrm{Mbp}$, and around 54.1 Mbp. Focus for the next section will be on the region around $51.3 \mathrm{Mbp}$ because this region contained the most significant SNP.

Focusing on the most significant region around 51.3 Mbp shows that 8 intronic SNP were located in the coiled-coil domain containing 57 (CCDC57) gene that were in perfect $\mathrm{LD}\left(\mathrm{r}^{2}=1\right)$ with each other and were the most significant SNP $\left[-\log _{10}(P\right.$-value $)=32.8$; Figure 1 and Table 1]. These SNP showed an allele substitution effect of $0.47 \mathrm{wt} / \mathrm{wt} \%$ (Table 1) and explained $21.6 \%$ of the total additive genetic variation in C14:0. The next most significant SNP [BovineHD1900014350; - $\log _{10}$
$(P$-value $)=31.4 ;$ Table 1$]$ was also located in $C C D C 5 \%$, and the $\mathrm{r}^{2}$ between this SNP and the 8 most significant SNP was 0.98 . This SNP was actually empirically genotyped and was the most significant SNP in previous GWAS (Bouwman et al., 2011, 2012). The next most significant SNP [BovineHD1900014372; $-\log _{10} \quad(P-$ value) $=31.2 ;$ Table 1$]$ was located in the FASN gene, which is adjacent to $C C D C 5 \%$, and the $\mathrm{r}^{2}$ between this SNP and the 8 most significant SNP was 0.40.

\section{2-SNP Window in FASN Most Significantly Associated With C14:0}

Screening BTA19 with a sliding window of 2 consecutive phased SNP, shifting 1 SNP at a time, resulted in the same chromosomal region showing the strongest signal as in the single SNP analysis (Figure 2), but
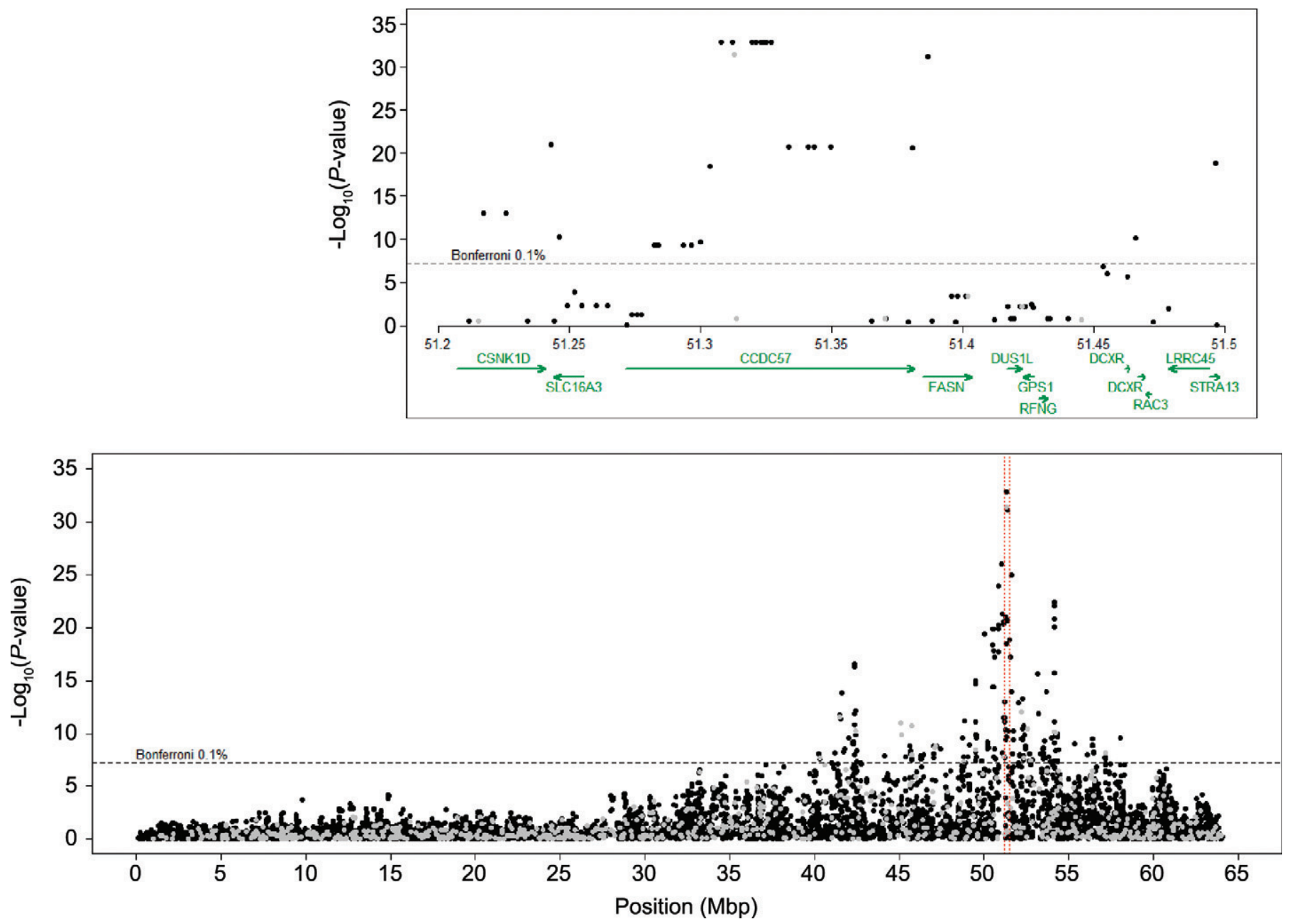

Figure 1. Manhattan plot of the single SNP association analysis of C14:0 on BTA19, with a zoom view of the region between 51.2 and 51.5 Mbp. Gray dots represent genotyped SNP, whereas black dots represent imputed SNP. All genes located in the region between 51.2 and 51.5 $\mathrm{Mbp}$ are represented by the green arrows in the zoom view. The dotted (red) vertical lines indicate the zoom view area. Color version available in the online PDF. 
Table 1. Details of the 10 most significant SNP from a single SNP association analysis of C14:0 on BTA19

\begin{tabular}{|c|c|c|c|c|c|c|c|}
\hline SNP name & $\begin{array}{l}\text { Position } \\
\text { (bp) }\end{array}$ & $\mathrm{MAF}^{1}$ & $\begin{array}{c}-\log _{10} \\
(P \text {-value })^{2}\end{array}$ & $\begin{array}{c}\text { Allele } \\
\text { substitution } \\
\text { effect }^{3}\end{array}$ & $\mathrm{SE}$ & Gene $^{4}$ & $\begin{array}{l}\text { Functional } \\
\text { consequence }\end{array}$ \\
\hline BovineHD1900014348 & $51,307,827$ & 0.45 & 32.8 & 0.47 & 0.04 & $C C D C 57$ & Intron \\
\hline BovineHD1900014354 & $51,319,695$ & 0.45 & 32.8 & 0.47 & 0.04 & $C C D C 57$ & Intron \\
\hline BovineHD1900014355 & $51,320,976$ & 0.45 & 32.8 & 0.47 & 0.04 & $C C D C 57$ & Intron \\
\hline BovineHD1900014356 & $51,322,876$ & 0.45 & 32.8 & 0.47 & 0.04 & $C C D C 57$ & Intron \\
\hline BovineHD1900014350 & $51,312,889$ & 0.44 & 31.4 & 0.47 & 0.04 & $C C D C 57$ & Intron \\
\hline BovineHD1900014372 & $51,386,738$ & 0.34 & 31.2 & -0.42 & 0.03 & FASN & Intron \\
\hline
\end{tabular}

${ }^{1}$ Minor allele frequency.

${ }^{2}$ The $-\log _{10}$ of the $P$-value from the association of the SNP with C14:0.

${ }^{3}$ The allele substitution effect was calculated using model 1 with a regression on the number of copies $(0,1$, or 2$)$ of the minor allele.

${ }^{4} C C D C 57=$ coiled-coil domain containing $57 ; F A S N=$ FA synthase.

focusing on the region around $51.3 \mathrm{Mbp}$ showed some small differences. The most significant window consisted of 2 SNP located in FASN (Table 2) instead of SNP in $C C D C 5 \%$. The other highly significant windows given in Table 2 consisted of 2 SNP located in CCDC57, except 1 window that consisted of the last SNP in $C C D C 5^{7} 7$ and the first SNP in FASN.

For the most significant window with both SNP located in FASN (BovineHD1900014372-BovineHD1900014373), a regression on the number of copies of each haplotype was performed. Haplotype G-G had a frequency of 0.48 and an allele substitution effect of 0.45 $( \pm 0.04)$; haplotype A-G had a frequency of 0.34 and an effect of $-0.42( \pm 0.03)$; haplotype G-A had a frequency of 0.18 and an effect of $0.05( \pm 0.04$; haplotype A-A was not present in the population studied). The allele substitution effects of the A-G and G-G haplotypes were of similar size compared with the allele substitution effect of BovineHD1900014372 from the single SNP analysis (Table 1).

\section{LD Block Contains Both CCDC57 and FASN}

Haploview analysis of the 84 SNP in the region between 51.2 and $51.5 \mathrm{Mbp}$ on BTA19 revealed $6 \mathrm{LD}$ blocks. Linkage disequilibrium block 3 (51,303,322-51,388,329 bp) contained $22 \mathrm{SNP}$, including the CCDC57 SNP that were most significant in the single SNP analysis and the 2 FASN SNP that were most significant in the sliding-window analysis. Therefore, LD block 3 was investigated in more detail. Within LD block 3, 9 different haplotypes had a frequency $>1 \%$ in the population (Table 3); HAPLO3 was the most frequent haplotype (0.368), followed by HAPLO2 (0.287; Table 4).

Association between C14:0 and the number of copies of the haplotype present per individual were analyzed for each of the 9 haplotypes. The haplotype HAPLO2 was most significantly associated with $\mathrm{C} 14: 0\left[-\log _{10}\right.$ $(P$-value $)=23.84]$, followed by HAPLO3 $\left[-\log _{10}(P\right.$ value $)=18.67$; Table 4]. The effects of HAPLO2 and HAPLO3 on C14:0 were similar but in opposite direc-

Table 2. Details of the 10 most significant 2-SNP windows associated with C14:0 on BTA19

\begin{tabular}{|c|c|c|c|c|c|}
\hline \multicolumn{2}{|c|}{ SNP 1} & \multicolumn{2}{|c|}{ SNP 2} & \multirow[b]{2}{*}{$\begin{array}{c}-\log _{10} \\
(P \text {-value })^{1}\end{array}$} & \multirow[b]{2}{*}{ Gene $^{2}$} \\
\hline SNP Name & $\begin{array}{l}\text { Position } \\
\text { (bp) }\end{array}$ & SNP Name & $\begin{array}{l}\text { Position } \\
\text { (bp) }\end{array}$ & & \\
\hline BovineHD1900014348 & $51,307,827$ & BovineHD1900014349 & $51,312,107$ & 31.8 & $C C D C 57$ \\
\hline BovineHD1900014354 & $51,319,695$ & BovineHD1900014355 & $51,320,976$ & 31.8 & $C C D C 57$ \\
\hline BovineHD1900014355 & $51,320,976$ & BovineHD1900014356 & $51,322,876$ & 31.8 & $C C D C 57$ \\
\hline BovineHD1900014358 & $51,325,153$ & ARS-BFGL-NGS-39328 & $51,326,752$ & 31.8 & $C C D C 57$ \\
\hline BovineHD1900014371 & $51,380,688$ & BovineHD1900014372 & $51,386,738$ & 30.8 & CCDC57/FASN \\
\hline BovineHD1900014349 & $51,312,107$ & BovineHD1900014350 & $51,312,889$ & 30.1 & $C C D C 57$ \\
\hline BovineHD1900014346 & $51,303,322$ & BovineHD1900014348 & $51,307,827$ & 29.0 & $C C D C 57$ \\
\hline
\end{tabular}

${ }^{1}$ The $-\log _{10}$ of the $P$-value from the association of the 2-SNP window with C14:0.

${ }^{2} F A S N=$ FA synthase; $C C D C 57=$ coiled-coil domain containing 57. 
tions: the regression coefficient of HAPLO2 was -0.37 $( \pm 0.03)$, whereas the regression coefficient of HAPLO3 was $0.35( \pm 0.04$; Table 4$)$. This agreed with the fact that HAPLO2 and HAPLO3 differed from each other at the SNP in LD block 3 that were significant in the single SNP analysis (Table 3). These haplotype allele substitution effects were a little smaller compared with the allele substitution effect of the most significant SNP in the single SNP analysis and the allele substitution effect of the most significant 2-SNP window in the sliding-window analysis. Therefore, the LD block haplotypes did not seem to capture more LD with the causal variant of the QTL compared with the single SNP and sliding-window analyses.

\section{2-SNP Window Captures Most of the QTL Variance}

Figure 3A shows the Manhattan plot of BTA19 after correction for the most significant SNP of the single-SNP association analysis (Bovine1900014354). The original single SNP analysis showed 3 significant regions: around 42.4, 51.3, and 54.1 Mbp. After correction for the most significant SNP located in CCDC57 (51.3 Mbp), the region around 42.2 Mbp dropped below the significance threshold, whereas the regions around 51.3 and 54.1 Mbp remained significant. The most significant SNP after correction (BovineHD1900018628) was located at 51,612,335 bp in the gene Aly/REF THO complex $4(\boldsymbol{A L} \boldsymbol{Y R} \boldsymbol{E F})$. The LD between this
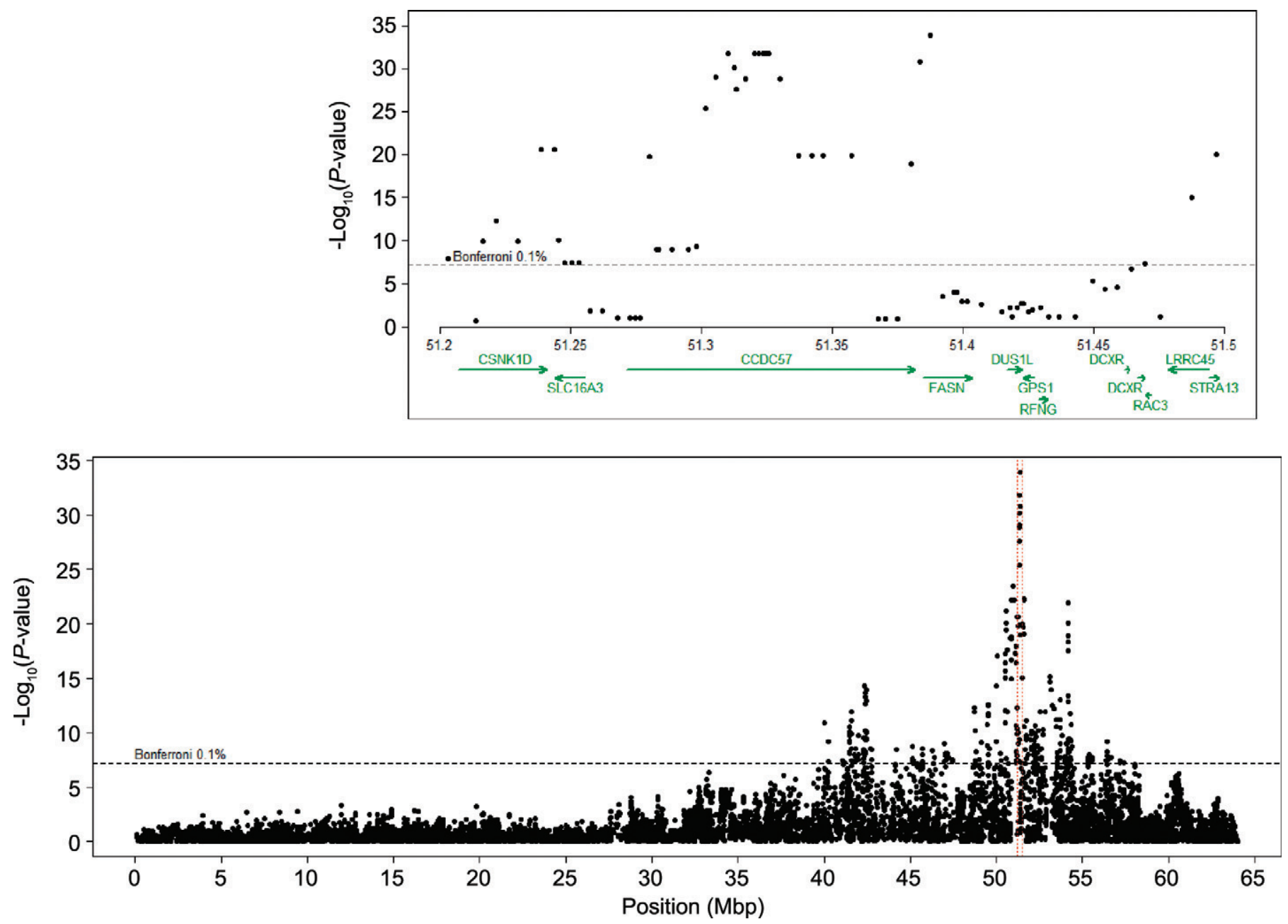

Figure 2. Manhattan plot of association analysis of C14:0 on BTA19 using a sliding window of 2 phased SNP, with a zoom view of the region between 51.2 and $51.5 \mathrm{Mbp}$. The marker positions in this figure were based on the average positions of the $2 \mathrm{SNP}$ in each window. All genes located in the region between 51.2 and $51.5 \mathrm{Mbp}$ are represented by the green arrows in the zoom view. The dotted (red) vertical lines indicate the zoom view area. Color version available in the online PDF. 


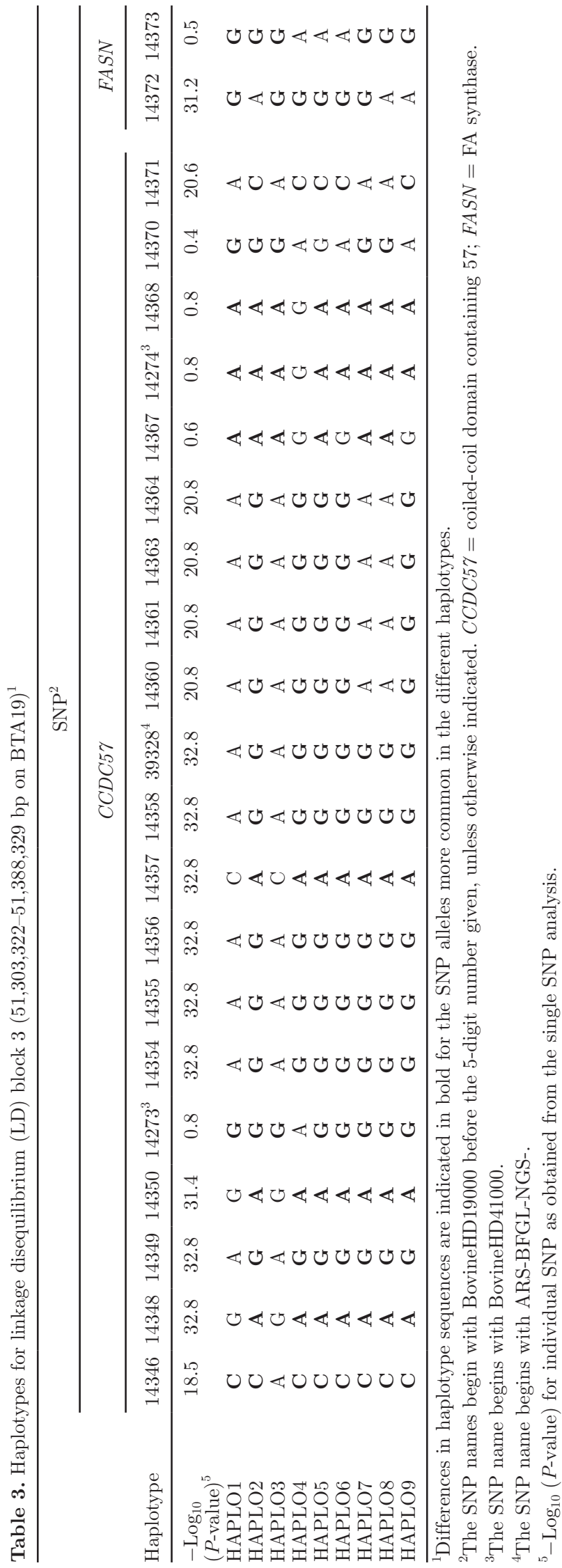

SNP in ALYREF and the most significant SNP from the original single SNP analysis was 0.17 . The next 5 most significant SNP after correction were located between $54,144,924$ and $54,166,828$ bp in the gene ubiquitin-specific peptidase 36 (USP36), of which one (BovineHD1900015164) was a missense mutation, causing an alanine to aspartic acid amino acid change. The LD between the most significant SNP in USP36 and the most significant SNP from the original single SNP analysis was 0.19 , whereas the LD between this USP36 SNP and the SNP in ALYREF was 0.43. The significance level of all other SNP significant in the original single SNP analysis dropped below the Bonferroni 0.1\% threshold after correction for the most significant SNP.

Figure 3B shows the Manhattan plot of BTA19 after correction for the most significant 2-SNP window (Bovine1900014372-Bovine1900014373) of the sliding-window analysis. After correction for the most significant 2-SNP window located in FASN (51.3 Mbp), all SNP significant in the original single SNP analysis dropped below the Bonferroni $0.1 \%$ threshold. This indicates that the 2-SNP window in FASN captured most of the QTL variance.

\section{DISCUSSION}

This study aimed to fine map a QTL for C14:0 content in bovine milk fat on BTA19. The previously identified QTL spanned a rather large region of almost half the chromosome (Bouwman et al., 2011). Using 10 times more SNP in the present study gave a more detailed view of the associated region and showed that the most significant SNP were located in an LD block that contained 2 genes: $C C D C 57$ and FASN.

\section{Fine Mapping Using Imputed SNP Data}

The QTL on BTA19 was fine mapped using genotypes imputed from a $50 \mathrm{~K}$ SNP panel to a $777 \mathrm{~K}$ SNP panel. Fine mapping the QTL on BTA19 using genotypes imputed from the 50K SNP panel to the $777 \mathrm{~K}$ SNP panel recovered the same region as being most significant compared with a previous $50 \mathrm{~K}$ genome-wide analysis (Bouwman et al. 2011) However, in the previous study, it could be suggested that SNP in CCDC57 picked up the effect of FASN, but now due to the higher density of SNP, we see that SNP in both genes are significantly associated with C14:0 and LD between SNP in the 2 genes is not high.

High-density SNP panels and sequence data will enhance QTL fine mapping and detection of causal variants. The $50 \mathrm{~K}$ SNP panel has a useful density to screen the genome for such QTL, whereas the higherdensity SNP panels are very useful to fine map the 
Table 4. Frequencies, significance levels, and allele substitution effects of haplotypes for linkage disequilibrium (LD) block 3 (51,303,322-51,388,329 bp on BTA19) associated with C14:0

\begin{tabular}{lcccc}
\hline Haplotype & Frequency & $\begin{array}{c}\text { Allele } \\
-\log _{10} \\
(P \text {-value })\end{array}$ & $\begin{array}{c}\text { substitution } \\
\text { effect }\end{array}$ & SE \\
\hline HAPLO1 & 0.073 & 5.27 & 0.28 & 0.06 \\
HAPLO2 & 0.287 & 23.84 & -0.37 & 0.03 \\
HAPLO3 & 0.368 & 18.67 & 0.35 & 0.04 \\
HAPLO4 & 0.083 & 1.18 & -0.11 & 0.06 \\
HAPLO5 & 0.051 & 2.14 & 0.12 & 0.08 \\
HAPLO6 & 0.049 & 1.01 & -0.06 & 0.08 \\
HAPLO7 & 0.035 & 0.29 & -0.45 & 0.10 \\
HAPLO8 & 0.023 & 4.96 & -0.11 & 0.11 \\
HAPLO9 & 0.019 & 0.48 & & \\
\hline
\end{tabular}
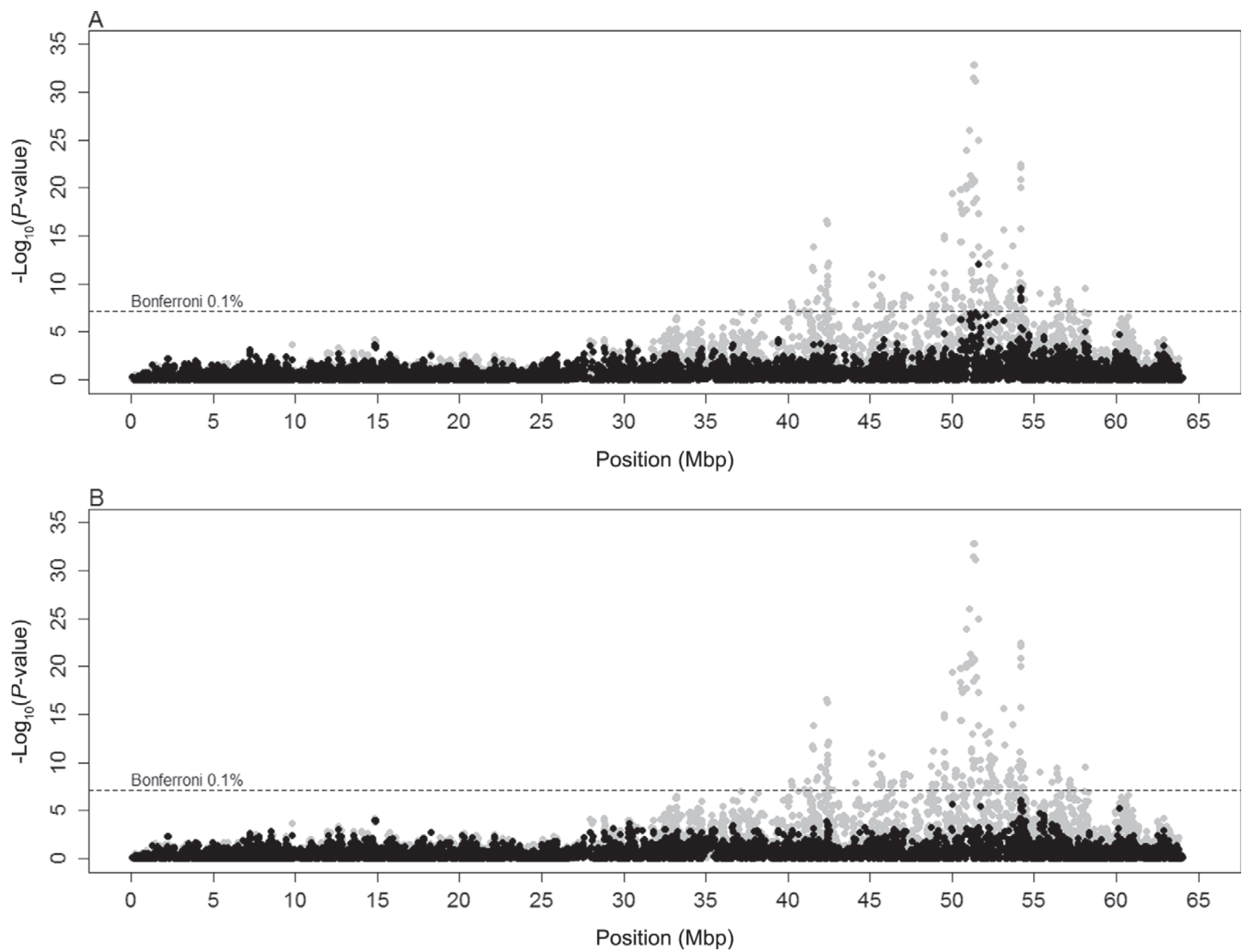

Figure 3. Manhattan plot of single SNP association analysis of C14:0 on BTA19 after correction for the effect of the most significant SNP of the single SNP analysis (A) and after correction for the most significant 2-SNP window of the sliding window analysis (B). In gray are the original single SNP analysis results. 
regions detected using the 50K SNP panels. Fine mapping using sequence data could even lead to detection of candidate causal variants because the causal variant should be present as a SNP in the sequence data of a population that is segregating for the QTL. However, functional studies are required to declare a candidate SNP as causal variant.

The numbers of animals with HD genotypes and especially with sequence data are still limited. Imputation of genotypes can, therefore, be a useful tool to increase the density of genotypes. Also, imputation is useful to facilitate combining genotype data generated with different genotyping arrays. In human studies, it has been shown that the power of GWAS increases when imputation is used to increase the number of genotypes (Marchini et al., 2007; Guan and Stephens, 2008; Spencer et al., 2009; Marchini and Howie, 2010). Small changes in imputation accuracy lead to small changes in power of GWAS; also, poor imputation accuracy can still improve power compared with no imputation (Guan and Stephens, 2008).

On the other hand Almeida et al. (2011) showed that the type-I error of association is higher for imputed SNP compared with empirically genotyped SNP; especially SNP with minor allele frequency close to 0.5 were false-positively associated. In the current study, one of the highly significant SNP in the studied LD block was genotyped (BovineHD1900014350), and that SNP was also the most significant SNP for C14:0 on BTA19 in Bouwman et al. (2011, 2012). In addition, a GWAS (50K) in beef cattle showed that 1 of the 8 most significant SNP (ARS-BFGL-NGS-39328) was most significantly associated with $\mathrm{C} 14: 0$ in adipose tissue (Ishii et al., 2013), indicating that it is unlikely that the detected associations with SNP in the haplotype block were false-positive associations.

Imputation accuracy depends on many factors, such as LD between SNP, allele frequency, number of animals genotyped with high density, and relationships between reference population and target population. Schrooten et al. (2012) showed that only 0.55 to $0.76 \%$ imputation errors were made using BEAGLE to impute individuals genotyped with 50K SNP panels to HD panels using 488 HD genotyped individuals. Because the HD genotyped reference population was much larger in the current study (1,333 individuals) and included the 55 sires of the imputed cows, it can be assumed that imputation errors were even lower.

Imputation accuracy also depends on the quality of the genome build. Erbe et al. (2012) and Pausch et al. (2013) showed that certain regions on the genome have poor imputation accuracy. Erbe et al. (2012) suggested that this poor imputation accuracy could be due to mapping errors. Imputation accuracy improved after remapping problem regions based on LD (Erbe et al., 2012). According to the studies of Erbe et al. (2012) and Pausch et al. (2013) no indication existed of low imputation accuracy or mapping errors on BTA19 in Holstein and Fleckvieh, respectively.

\section{CCDC57}

Fine-mapping the QTL for C14:0 content in bovine milk fat on BTA19 gave a more detailed view of the associated region and showed that the most significant SNP were located in an LD block that contained 2 genes: $C C D C 57$ and FASN. The gene CCDC57 is minimally characterized and has not been associated with bovine milk fat previously; it is transcribed into a coiled-coil domain containing protein. Coiled-coil domains are structural motifs in proteins, with many involved in important biological functions such as DNA binding and regulation of gene expression. The gene CCDC57 is located next to FASN, which is a more pronounced candidate gene because of its known biological relation to fat synthesis. However, the candidacy of $C C D C 5^{7}$ is supported by Medrano et al. (2010), who showed that $C C D C 57$ was expressed in mammary tissue of a secondlactation cow and that the expression level of $C C D C 57$ was higher than that of FASN.

\section{FASN}

In addition to $C C D C 5 \%$, the current study also suggested FASN as a candidate gene, because, besides SNP in $C C D C 5 \%$, a 2-SNP window located in FASN also was highly significantly associated with C14:0 content. The gene FASN encodes a multi-enzyme system that catalyzes de novo FA synthesis. Even though $F A S N$ has been studied extensively in candidate gene studies for fat content, milk FA, and FA in adipose tissue (Roy et al., 2006; Zhang et al., 2008; Abe et al., 2009; Schennink et al., 2009a; Li et al., 2012; Oh et al., 2012), this has not yet resulted in identification of the causal variant for the QTL on BTA19.

Three SNP on the HD SNP panel located in FASN have been associated with milk FA in dairy cattle and with FA in adipose tissue in beef cattle: BovineHD1900014375 (g.13126C > T; position relative to the sequence of FASN with accession AF285607), BovineHD1900014377 (g.16907T $>$ C), and BovineHD1900014275 (g.17924A>G; Morris et al., 2007; Zhang et al., 2008; Schennink et al., 2009a; Li et al., 2012; Oh et al., 2012). A fourth SNP on the HD SNP panel located in FASN, BovineHD1900014376 (g.13965C > T), was detected by Abe et al. (2009) but not studied in detail. The current study showed that associations with these $4 \mathrm{SNP}$ were relatively weak $\left[-\log _{10}\right.$ ( $P$-values $)$ 
were $0.4,3.4,21.3$, and 3.4 , respectively] compared with the considerably stronger association that was found with another FASN SNP BovineHD1900014372 [- $\log _{10}$ $(P$-value $)=31.2]$.

\section{Other Fat Synthesis-Related Genes}

In addition to $C C D C 57$ and $F A S N$, additional candidate genes known to be involved in milk fat synthesis underlie the QTL on BTA19, such as ATP citrate lyase $(A C L Y)$, sterol regulatory element-binding transcription factor 1 (SREBF1), signal transducer and activator of transcription 5A (STAT5A), and growth hormone 1 (GH1). Of the $8 \mathrm{SNP}$ in $A C L Y, 1 \mathrm{SNP}\left[-\log _{10}(P-\right.$ value $)=7.18]$ just exceeded the Bonferroni threshold and $3 \mathrm{SNP}\left[-\log _{10}(P\right.$-value $\left.)=7.13\right]$ were just below that threshold, but they reduced considerably in significance when corrected for the effect of the most significant SNP or 2-SNP window (Figure 3). The 2 SNP in our HD SNP panel located in SREBF1 and 4 SNP located in STAT5A did not show any association with C14:0. The only SNP located in GH1 was below the Bonferroni threshold $\left[-\log _{10}(P\right.$-value $\left.)=6.82\right]$, and also reduced considerably in significance when corrected for the effect of the most significant SNP or 2-SNP window (Figure 3).

\section{CONCLUSIONS}

We reduced the QTL for C14:0 on BTA19 to an LD block formed by 22 SNP covering 85,007 bp (51,303,322-51,388,329 bp). This LD block contained 2 genes: $C C D C 57$ and $F A S N$. The gene $C C D C 57$ is minimally characterized and has not been associated with bovine milk fat previously, but is expressed in the mammary gland. In addition to SNP located in $C C D C 5 \%$, a 2-SNP window located in FASN also was highly significantly associated with $\mathrm{C} 14: 0$ content. The gene $F A S N$ is involved in de novo fat synthesis and has been studied in candidate gene studies. Future studies using sequence data of both CCDC57 and FASN and, eventually, functional studies will have to be pursued to assign the causal variant(s).

\section{ACKNOWLEDGMENTS}

Chris Schrooten [Cooperative Cattle Improvement Organization (CRV), Arnhem, the Netherlands] is acknowledged for the imputation of the genotypes. This study is part of the Dutch Milk Genomics Initiative and the project "Melk op Maat," funded by Wageningen University (Wageningen, the Netherlands), the Dutch Dairy Association (NZO, Zoetermeer, the Netherlands), CRV, the Dutch Technology Foundation
(STW, Utrecht, the Netherlands), the Dutch Ministry of Economic Affairs (The Hague, the Netherlands), and the Provinces of Gelderland and Overijssel (Arnhem, the Netherlands).

\section{REFERENCES}

Abe, T., J. Saburi, H. Hasebe, T. Nakagawa, S. Misumi, T. Nade, H. Nakajima, N. Shoji, M. Kobayashi, and E. Kobayashi. 2009. Novel mutations of the FASN gene and their effect on fatty acid composition in Japanese Black beef. Biochem. Genet. 47:397-411.

Almeida, M. A. A., P. S. L. Oliveira, T. V. Pereira, J. E. Krieger, and A. C. Pereira. 2011. An empirical evaluation of imputation accuracy for association statistics reveals increased type-I error rates in genome-wide associations. BMC Genet. 12:10.

Barrett, J. C., B. Fry, J. Maller, and M. J. Daly. 2005. Haploview: Analysis and visualization of LD and haplotype maps. Bioinformatics 21:263-265.

Blott, S., J.-J. Kim, S. Moisio, A. Schmidt-Küntzel, A. Cornet, P. Berzi, N. Cambisano, C. Ford, B. Grisart, D. Johnson, L. Karim, P. Simon, R. Snell, R. Spelman, J. Wong, J. Vilkki, M. Georges, F. Farnir, and W. Coppieters. 2003. Molecular dissection of a quantitative trait locus: A phenylalanine-to-tyrosine substitution in the transmembrane domain of the bovine growth hormone receptor is associated with a major effect on milk yield and composition. Genetics 163:253-266.

Bouwman, A. C., H. Bovenhuis, M. H. P. W. Visker, and J. A. M. van Arendonk. 2011. Genome-wide association of milk fatty acids in Dutch dairy cattle. BMC Genet. 12:43.

Bouwman, A. C., M. H. P. W. Visker, J. A. M. van Arendonk, and H. Bovenhuis. 2012. Genomic regions associated with bovine milk fatty acids in both summer and winter milk samples. BMC Genet. 13:93.

Browning, B. L., and S. R. Browning. 2009. A unified approach to genotype imputation and haplotype-phase inference for large data sets of trios and unrelated individuals. Am. J. Hum. Genet. 84:210-223.

Cohen-Zinder, M., E. Seroussi, D. M. Larkin, J. J. Loor, A. E.-d. Wind, J.-H. Lee, J. K. Drackley, M. R. Band, A. G. Hernandez, M. Shani, H. A. Lewin, J. I. Weller, and M. Ron. 2005. Identification of a missense mutation in the bovine $A B C G 2$ gene with a major effect on the QTL on chromosome 6 affecting milk yield and composition in Holstein cattle. Genome Res. 15:936-944.

Druet, T., S. Fritz, M. Boussaha, S. Ben-Jemaa, F. Guillaume, D. Derbala, D. Zelenika, D. Lechner, C. Charon, D. Boichard, I. G. Gut, A. Eggen, and M. Gautier. 2008. Fine mapping of quantitative trait loci affecting female fertility in dairy cattle on BTA03 using a dense single-nucleotide polymorphism map. Genetics 178:2227-2235.

Durinck, S., P. T. Spellman, E. Birney, and W. Huber. 2009. Mapping identifiers for the integration of genomic datasets with the R/Bioconductor package biomaRt. Nat. Protoc. 4:1184-1191.

Erbe, M., B. J. Hayes, L. K. Matukumalli, S. Goswami, P. J. Bowman, C. M. Reich, B. A. Mason, and M. E. Goddard. 2012. Improving accuracy of genomic predictions within and between dairy cattle breeds with imputed high-density single nucleotide polymorphism panels. J. Dairy Sci. 95:4114-4129.

Gautier, M., R. R. Barcelona, S. Fritz, C. Grohs, T. Druet, D. Boichard, A. Eggen, and T. H. E. Meuwissen. 2006. Fine mapping and physical characterization of two linked quantitative trait loci affecting milk fat yield in dairy cattle on BTA26. Genetics 172:425-436.

Grisart, B., W. Coppieters, F. Farnir, L. Karim, C. Ford, P. Berzi, N. Cambisano, M. Mni, S. Reid, P. Simon, R. Spelman, M. Georges, and R. Snell. 2002. Positional candidate cloning of a QTL in dairy cattle: Identification of a missense mutation in the bovine DGAT1 gene with major effect on milk yield and composition. Genome Res. 12:222-231.

Guan, Y., and M. Stephens. 2008. Practical issues in imputation-based association mapping. PLoS Genet. 4:e1000279. 
Hu, Z.-L., C. A. Park, E. R. Fritz, and J. M. Reecy. 2010. QTLdb: A comprehensive database tool building bridges between genotypes and phenotypes. Page 137 in Proc. of the 9th World Congr. Genet. Appl. Livest. Prod., Leipzig, Germany. German Society for Animal Science, Neustadt, Germany

Ishii, A., K. Yamaji, Y. Uemoto, N. Sasago, E. Kobayashi, N. Kobayashi, T. Matsuhashi, S. Maruyama, H. Matsumoto, S. Sasazaki, and H. Mannen. 2013. Genome-wide association study for fatty acid composition in Japanese Black cattle. Anim. Sci. J. 84:675-682.

Karim, L., H. Takeda, L. Lin, T. Druet, J. A. C. Arias, D. Baurain, N. Cambisano, S. R. Davis, F. Farnir, B. Grisart, B. L. Harris, M. D. Keehan, M. D. Littlejohn, R. J. Spelman, M. Georges, and W. Coppieters. 2011. Variants modulating the expression of a chromosome domain encompassing PLAG1 influence bovine stature. Nat. Genet. 43:405-413.

Khatkar, M. S., P. C. Thomson, I. Tammen, and H. W. Raadsma 2004. Quantitative trait loci mapping in dairy cattle: Review and meta-analysis. Genet. Sel. Evol. 36:163-190.

Kim, E.-S., X. Shi, O. Cobanoglu, K. Weigel, P. J. Berger, and B. W. Kirkpatrick. 2009. Refined mapping of twinning-rate quantitative trait loci on bovine chromosome 5 and analysis of insulinlike growth factor-1 as a positional candidate gene. J. Anim. Sci. 87:835-843.

Li, C., N. Aldai, M. Vinsky, M. E. R. Dugan, and T. A. McAllister. 2012. Association analyses of single nucleotide polymorphisms in bovine stearoyl-CoA desaturase and fatty acid synthase genes with fatty acid composition in commercial cross-bred beef steers. Anim. Genet. 43:93-97.

Marchini, J., and B. Howie. 2010. Genotype imputation for genomewide association studies. Nat. Rev. Genet. 11:499-511.

Marchini, J., B. Howie, S. Myers, G. McVean, and P. Donnelly. 2007. A new multipoint method for genome-wide association studies by imputation of genotypes. Nat. Genet. 39:906-913.

Medrano, J., G. Rincon, and A. Islas-Trejo. 2010. Comparative analysis of bovine milk and mammary gland transcriptome using RNASeq. Page 125 in Proc. 9th World Congr. Genet. Appl. Livest. Prod., Leipzig, Germany. German Society for Animal Science, Neustadt, Germany.

Meuwissen, T. H. E., A. Karlsen, S. Lien, I. Olsaker, and M. E. Goddard. 2002. Fine mapping of a quantitative trait locus for twinning rate using combined linkage and linkage disequilibrium mapping. Genetics 161:373-379.

Morris, C. A., N. G. Cullen, B. C. Glass, D. L. Hyndman, T. R. Manley, S. M. Hickey, J. C. McEwan, W. S. Pitchford, C. D. K. Bottema, and M. A. H. Lee. 2007. Fatty acid synthase effects on bovine adipose fat and milk fat. Mamm. Genome 18:64-74.

Oh, D., Y. Lee, B. La, J. Yeo, E. Chung, Y. Kim, and C. Lee. 2012 Fatty acid composition of beef is associated with exonic nucleotide variants of the gene encoding FASN. Mol. Biol. Rep. 39:40834090.

Ordovás, L., R. Roy, S. Pampín, P. Zaragoza, R. Osta, J. C. Rodríguez-Rey, and C. Rodellar. 2008. The g.763G $>$ C SNP of the bovine FASN gene affects its promoter activity via Sp-mediated regulation: Implications for the bovine lactating mammary gland. Physiol. Genomics 34:144-148.
Pausch, H., B. Aigner, R. Emmerling, C. Edel, K.-U. Götz, and R. Fries. 2013. Imputation of high-density genotypes in the Fleckvieh cattle population. Genet. Sel. Evol. 45:3.

Roy, R., L. Ordovas, P. Zaragoza, A. Romero, C. Moreno, J. Altarriba, and C. Rodellar. 2006. Association of polymorphisms in the bovine FASN gene with milk-fat content. Anim. Genet. 37:215-218.

Schennink, A., H. Bovenhuis, K. M. Léon-Kloosterziel, J. A. M. Van Arendonk, and M. H. P. W. Visker. 2009a. Effect of polymorphisms in the FASN, OLR1, PPARGC1A, PRL and STAT5A genes on bovine milk-fat composition. Anim. Genet. 40:909-916.

Schennink, A., J. M. L. Heck, H. Bovenhuis, M. H. P. W. Visker, H. J. F. van Valenberg, and J. A. M. van Arendonk. 2008. Milk fatty acid unsaturation: Genetic parameters and effects of stearoyl-CoA desaturase $(S C D 1)$ and acyl CoA: diacylglycerol acyltransferase 1 (DGAT1). J. Dairy Sci. 91:2135-2143.

Schennink, A., W. M. Stoop, M. H. P. W. Visker, J. M. L. Heck, H. Bovenhuis, J. J. Van Der Poel, H. J. F. Van Valenberg, and J. A M. Van Arendonk. 2007. DGAT1 underlies large genetic variation in milk-fat composition of dairy cows. Anim. Genet. 38:467-473.

Schennink, A., W. M. Stoop, M. H. P. W. Visker, J. J. van der Poel, H. Bovenhuis, and J. A. M. van Arendonk. 2009b. Short communication: Genome-wide scan for bovine milk-fat composition. II. Quantitative trait loci for long-chain fatty acids. J. Dairy Sci. 92:4676-4682.

Schrooten, C., R. Dassonneville, R. Brøndum, J. Chen, Z. Liu, and T. Druet. 2012. Error rate for imputation from BovineSNP50 to BovineHD. Page 137 in Proc. 63rd Annual Meeting of the European Federation of Animal Science (EAAP), Bratislava, Slovakia. Wageningen Academic Publishers, Wageningen, the Netherlands.

Šidák, Z. 1967. Rectangular confidence regions for the means of multivariate normal distributions. J. Am. Stat. Assoc. 62:626-633.

Spencer, C. C. A., Z. Su, P. Donnelly, and J. Marchini. 2009. Designing genome-wide association studies: Sample size, power, imputation, and the choice of genotyping chip. PLoS Genet. 5:e1000477.

Stoop, W. M., A. Schennink, M. H. P. W. Visker, E. Mullaart, J. A. M. van Arendonk, and H. Bovenhuis. 2009. Genome-wide scan for bovine milk-fat composition. I. Quantitative trait loci for short- and medium-chain fatty acids. J. Dairy Sci. 92:4664-4675.

Stoop, W. M., J. A. M. van Arendonk, J. M. L. Heck, H. J. F. van Valenberg, and H. Bovenhuis. 2008. Genetic parameters for major milk fatty acids and milk production traits of Dutch HolsteinFriesians. J. Dairy Sci. 91:385-394.

Taniguchi, M., T. Utsugi, K. Oyama, H. Mannen, M. Kobayashi, Y. Tanabe, A. Ogino, and S. Tsuji. 2004. Genotype of stearoyl-CoA desaturase is associated with fatty acid composition in Japanese Black cattle. Mamm. Genome 15:142-148.

Zhang. S., T. J. Knight, J. M. Reecy, and D. C. Beitz. 2008. DNA polymorphisms in bovine fatty acid synthase are associated with beef fatty acid composition. Anim. Genet. 39:62-70.

Zimin, A. V., A. L. Delcher, L. Florea, D. R. Kelley, M. C. Schatz, D. Puiu, F. Hanrahan, G. Pertea, C. P. Van Tassell, T. S. Sonstegard, G. Marçais, M. Roberts, P. Subramanian, J. A. Yorke, and S. L. Salzberg. 2009. A whole-genome assembly of the domestic cow, Bos taurus. Genome Biol. 10:R42. 